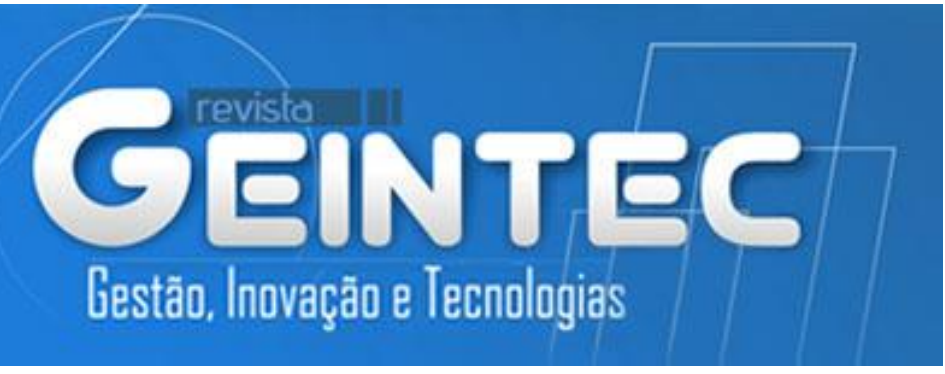

\title{
ANÁLISE DAS AÇÕES DO POLO DE EXCELÊNCIA EM FLORESTAS PARA A INOVAÇÃO E SUA CONTRIBUIÇÃO PARA A O SETOR FLORESTAL EM MINAS GERAIS
}

\section{ANALYSIS OF ACTIONS OF THE POLO DE EXCELÊNCIA EM FLORESTAS FOR INNOVATION AND ITS CONTRIBUTION TO THE FOREST SECTOR IN MINAS GERAIS}

\author{
Mariana Luísa da Costa Lage ${ }^{1}$; Rodrigo Gava ${ }^{2}$; Jeice Catrine Cordeiro Moreira ${ }^{3 ;}$ Afonso Augusto Teixeira de \\ Freitas de Carvalho Lima $^{4}$ \\ ${ }^{1}$ Programa de Pós-Graduação em Administração - PPGADM/UFES \\ Universidade Federal de Juiz de Fora - UFJF - Governador Valadares/MG - Brasil \\ marianaluisa@gmail.com \\ ${ }^{2}$ Programa de Pós-Graduação em Administração - PPGADM \\ Universidade Federal de Viçosa - UFV - Viçosa/MG - Brasil \\ rgava@ufv.br \\ ${ }^{3}$ Programa de Pós-Graduação em Controladoria e Contabilidade - PPGCC \\ Universidade de São Paulo - USP - São Paulo/SP - Brasil \\ jeicemoreira@gmail.com \\ ${ }^{4}$ Programa de Pós-Graduação em Administração - PPGADM \\ Universidade Federal de Viçosa - UFV - Viçosa/MG - Brasil \\ afonsoli@ufv.br
}

\begin{abstract}
Resumo
A inovação tecnológica assume destaque por manifestar-se como a mais importante força do crescimento econômico. $O$ objetivo deste estudo foi compreender as ações realizadas pelo Polo de Excelência em Florestas em busca da inovação no setor florestal de Minas Gerais. A teoria utilizada discorre sobre o Sistema de Inovação e a Hélice Tríplice, como fundamento para a articulação entre governo, universidade e setor privado na promoção da inovação. Trata-se de uma pesquisa descritiva realizada por meio de análise documental, observação e entrevistas semiestruturadas. Utilizou-se o modelo de processo de inovação de Bessant e Tidd (2009) para analisar em que níveis se concentravam as ações na busca pela inovação. Os resultados da pesquisa apontam que Polo de Excelência em Florestas desenvolveu, entre 2007 e 2013, essencialmente, projetos que buscam gerar inovação, em estágio primário, e quatro deles em estágio de seleção da inovação, em estágio secundário. Destaca-se o projeto Sistema de Informação Florestal, responsável pela criação do Centro de Inteligência Florestal (CIFlorestas), da Biblioteca Virtual Florestal e do Bureau de Inteligência Florestal, como resultados dos esforços do Polo em se tornar referência no setor florestal.
\end{abstract}

Palavras-chave: Políticas públicas, inovação, setor florestal.

Abstract 
The technological innovation has been receiving the spotlight due to manifest itself as the most important branch of the economic growth. The current study aimed to understand the actions of the Polo de Excelência em Florestas seeking of innovation in the forest sector of Minas Gerais. The theory used discusses the Innovation System and the Triple Helix as the basis for the relationship between government, academia and the private sector in promoting innovation. This is a descriptive study through document analysis, observation and semi-structured interviews. It was used the Bessant and Tidd (2009) innovation process model to analyze the levels were concentrated innovation actions. The research results reveal that Polo de Excelência em Florestas developed between 2007 and 2013, essentially projects that seek to generate innovation in primary stage, and four of them on stage selection of innovation in secondary stage. It was identified the Sistema de Informação Florestal project, responsible for the creation of the Centro de Inteligência Florestal (CIFlorestas), Biblioteca Virtual Florestal and the Bureau de Inteligência Florestal as results of Polo's efforts to become a leader in the forest sector.

Key-words: Public policy, innovation, forest sector.

\section{Introdução}

A inovação tecnológica assume destaque por manifestar-se como a mais importante força do crescimento econômico. Ao focar nas características mais distintivas da inovação nas economias de alto nível de industrialização, e nas que as pretendem ser, a força da relação entre a inovação e o movimento de produção-consumo conferiu-lhes condição de gêmeos inseparáveis na economia contemporânea (ROSENBERG, 2004). Simione, Binotto e Hoff (2012) destacam a importância das inovações tecnológicas na indústria da madeira, sobretudo por considerar a densidade tecnológica do setor.

Diversos autores defendem a ideia de que é impossível um ator inovar isoladamente, uma vez que a inovação é um processo dinâmico e colaborativo (BESSANT; TIDD, 2009; CASSIOLATO; LASTRES, 2005; EDQUIST, 2006; FREEMAN, 1995). Portanto, o termo inovação tecnológica muda de sentido, incorporando aspectos ambientais, políticos, sociais e culturais, além dos de mercado.

A partir desse cenário, surge o conceito de Sistema de Inovação (SI), caracterizado por:

\footnotetext{
...um conjunto de instituições distintas que contribuem para o desenvolvimento da capacidade de inovação e aprendizado de um país, região, setor ou localidade - e também o afetam. Constituem-se de elementos e relações que interagem na produção, difusão e uso do conhecimento... (CASSIOLATO; LASTRES, 2005, p. 37).
}

Assim, as abordagens sistêmicas da inovação valorizam a interação das instituições tanto na criação do conhecimento como em sua difusão e aplicação, enfatizando a importância das políticas de inovação.

A Hélice Tríplice, um modelo de sistema de inovação, pressupõe que somente a interação entre governo-universidade-indústria pode propiciar "um sistema de inovação sustentável e durável na era da economia do conhecimento" (VALENTE, 2010, p. 6).

A atuação em regime de Hélice Tríplice inicia em contexto regional quando os três atores universidade (desenvolvimento acadêmico), empresas (clusters industriais) e governo - iniciam um 
relacionamento recíproco, tentando melhorar um o desempenho do outro (ETZKOWITZ, 2009). Embora eles tenham interesses específicos, suas ações se complementam no fortalecimento do sistema de inovação (ETZKOWITZ, 2002)

O governo pode assumir papel importante na difusão da inovação ao incentivar as interações entre a universidade e o setor privado de várias maneiras. De acordo com Etzkowitz (2009), o governo desempenha um papel fundamental como um agente de risco do recurso público, por meio de programas em níveis federal e estadual para preencher a lacuna entre o apoio universitário na formação de empresas e a aceitação por apoiadores financeiros de capital privado de risco. Em relação aos investimentos, quanto mais avançada for a ideia técnica, maior a possibilidade de conseguir um aporte de dinheiro por parte do governo (ETZKOWITZ, 2009). Esse recurso pode ir tanto para o mercado, quanto para a pesquisa, proporcionando uma oportunidade de alcançar o desenvolvimento da inovação.

O Governo de Minas Gerais, após transformações nas configurações políticas e de gestão e o reconhecimento da existência de um paradigma tecnológico, criou uma série de iniciativas na área de ciência e tecnologia com o intuito de se desenvolver economicamente e se inserir na economia baseada no conhecimento. Nesse contexto, criaram-se, em 2007, os Polos de Excelência, de responsabilidade da Secretaria de Estado de Ciência, Tecnologia e Ensino Superior (SECTES). Trata-se de um termo de cooperação técnica entre atores - governo, setor privado, associações e instituições de ensino e pesquisa - diretamente envolvidos com a organização e o fortalecimento das estruturas geradoras de conhecimento, tecnologias, formação de recursos humanos e prestação de serviços para a promoção do desenvolvimento setorial (MINAS GERAIS, 2010).

Para isso, foram escolhidos setores estratégicos nos quais Minas Gerais tem tradição, massa crítica ou vantagens competitivas, como o setor florestal. Vale ressaltar que, embora os Polos estejam geograficamente localizados em determinadas cidades - neste caso Viçosa, com sede na Universidade Federal de Viçosa (UFV), eles atuam em outras regiões do estado e do país, assim como em nível internacional, sendo incentivados a buscar parcerias.

Nesse sentido, buscar-se-á a resposta para a seguinte questão: como se desenvolveram as ações propostas pelo Polo de Excelência em Florestas com vistas a produzir a inovação no setor florestal e, por conseguinte, para o estado de Minas Gerais?

\section{MATERIAL E MÉTODOS}

Em relação à coleta de dados, procuraram-se evidências de diversas fontes, primárias e secundárias. Os dados secundários foram coletados a partir da análise de documentos, e os dados primários, por meio de entrevistas semiestruturadas e observação. Destaca-se que o estudo revela ações desde a criação do Polo em 2007 até o ano de 2013. 
Com o intuito de analisar o processo de inovação, adotaram-se os conceitos e modelos de Bessant e Tidd (2009). Para eles, as inovações devem ser compreendidas como uma sequência estendida de atividades, ou seja, um processo. Portanto, "a inovação é a sequência de tais atividades envolvidas no processo de tornar uma ideia, ou possibilidade, realidade" (BESSANT; TIDD, 2009, p. 33).

A Figura 1 exemplifica o processo básico e mínimo para assegurar que a inovação ocorra. No primeiro momento, pautado pela geração de possibilidades de inovação, devem-se compreender os cenários, buscando examinar e analisar, com os objetivos de captar e processar sinais potenciais sobre inovação. Estes, por exemplo, podem surgir a partir de pressões regulatórias e oportunidades de pesquisas (BESSANT; TIDD, 2009).

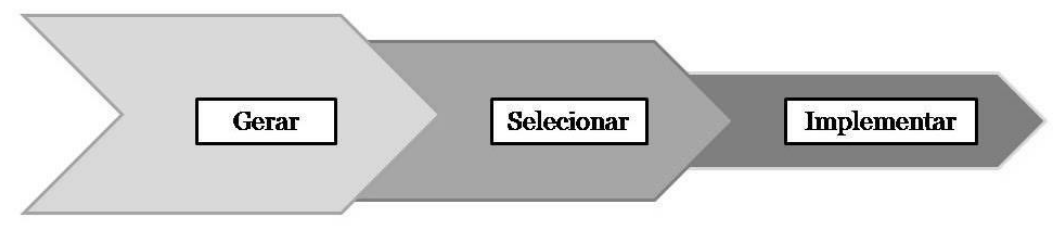

Figura 1 - Processo de Inovação

Fonte: BESSANT; TIDD (2009)

A seleção estratégica compõe o segundo momento. A partir dos sinais identificados no passo anterior, é preciso selecionar o que deve ser investido, analisando o potencial inovador e os recursos necessários, a fim de que isso gere vantagem competitiva para a organização (BESSANT; TIDD, 2009).

Finalizando, segue a implementação, tornando a inovação uma realidade, seja um produto/serviço, um novo processo ou um novo método. Ressalta-se que esta fase possui certo nível de dificuldade considerável, uma vez que o cenário é de incertezas, e ainda, surge uma série de problemas, como prazos e orçamentos, recursos humanos capacitados e com as habilidades essenciais, imperfeições, entre outros (BESSANT; TIDD, 2009).

\section{RESULTADOS}

Os Polos de Excelência são “ambientes adequados para investimentos de alto valor agregado e de referência nacional e internacional que integram competências institucionais para induzir o processo e desenvolvimento sustentável de cada setor" (MINAS GERAIS, 2010, p. 54).

O Polo de Excelência em Florestas configura-se por meio de um arranjo organizacional, que é constituído e inserido em uma região da Zona da Mara de Minas Gerais por reunir diversos fatores e atores ligados ao setor ou cadeia produtiva de base florestal (MINAS GERAIS, 2010). 
Os atores que participam deste termo de cooperação técnica e constituem o Polo de Excelência em Florestas são:

1. Instituições de Ensino Superior: Universidade Federal de Viçosa - UFV; Universidade Federal de Lavras - UFLA; Universidade Federal dos Vales do Jequitinhonha e Mucuri UFVJM.

2. Secretarias de Estado: Secretaria de Estado de Agricultura, Pecuária e Abastecimento SEAPA; Secretaria de Estado de Desenvolvimento Econômico - SEDE; Secretaria de Estado de Ciência, Tecnologia e Ensino Superior - SECTES; Secretaria de Estado de Meio Ambiente e Desenvolvimento Sustentável - SEMAD.

3. Entidades de Classe: Federação da Agricultura e Pecuária do Estado de Minas Gerais FAEMG; Sindicato Intermunicipal das Indústrias de Marcenaria de Ubá - INTERSIND.

4. Instituição de Apoio às Micro e Pequenas Empresas: Serviço de Apoio às Micro e Pequenas Empresas de Minas Gerais - SEBRAE/MG.

5. Organizações do Setor Produtivo: Associação Mineira de Silvicultura - AMS; Sociedade de Investigações Florestais - SIF.

6. Centro de Pesquisa: Embrapa Florestas - Unidade da Empresa Brasileira de Pesquisa Agropecuária localizada no estado do Paraná; Empresa de Pesquisa Agropecuária de Minas Gerais - EPAMIG.

A proposta é que o Polo de Excelência em Florestas atue como um catalisador de demandas entre o mercado e as instituições de ensino e pesquisa, buscando inovações. Ao executar essa articulação, o Polo contribui para o Sistema Agroindustrial de Base Florestal e busca ser referência nos cenários nacional e internacional.

Com o intuito de melhor compreender as ações desenvolvidas pelo Polo de Excelência em Florestas em busca da inovação setorial, optou-se por dividi-las em quatro:

- Projetos desenvolvidos: inclui todos os projetos do Polo de Excelência em Florestas que já foram concluídos e os que estão em andamento.

- Projetos propostos: lista os projetos do Polo de Excelência em Florestas que foram submetidos à SECTES e que aguardavam aprovação.

- Possíveis projetos: lista as possibilidades de novos projetos a serem submetidos à SECTES.

- Publicações do Polo de Excelência em Florestas: lista todas as publicações do Polo de Florestas até 2013.

Desde a criação do Polo foram desenvolvidas 110 ações (Tabela 1) e investidos $\mathrm{R} \$ 11.371 .844,33$ em projetos desenvolvidos.

Vale destacar que o Polo em si não é o desenvolvedor dos projetos, cabendo-lhe atuar como intermediador de demandas entre atores, elaborar os projetos e depois gerenciá-los. 
Tabela 1 - Ações desenvolvidas pelo Polo de Excelência em Florestas

\begin{tabular}{cc}
\hline Atividades & Quantidade \\
\hline Projetos Desenvolvidos & 21 \\
Projetos Propostos & 13 \\
Possíveis Projetos & 7 \\
Publicações & 69 \\
\hline Total & $\mathbf{1 1 0}$ \\
\hline
\end{tabular}

Fonte: Dados da pesquisa (2014).

Ao analisar os projetos (desenvolvidos, propostos e possíveis) optou-se por agrupá-los em: projetos de gestão do Polo; de sistema de informação; de pesquisas em geral; e de pesquisa específica com enfoque em inovação.

\subsection{Projetos desenvolvidos}

\subsubsection{Projetos de gestão do Polo de Excelência em Florestas}

Desde 2007, quatro projetos foram criados e cerca de $\mathrm{R} \$ 3.731 .095,01$ foram gastos na consolidação e gestão do Polo de Excelência em Florestas:

- Projeto Especial de Implantação do Polo de Excelência em Florestas.

- Consolidação dos Polos de Excelência em Minas Gerais - Projeto INOVAMINAS.

- Programa de Manutenção e Consolidação do Polo de Excelência em Florestas.

- Programa de Gestão do Polo de Excelência em Florestas.

O primeiro deles foi criado para subsidiar o início do funcionamento do Polo, antes mesmo de sua inauguração, em 2008. Nesse período se deu a elaboração de um Programa de Gestão, reuniões e a compra de equipamentos para criação de uma infraestrutura de funcionamento mesmo que em sede provisória, no Departamento de Engenharia Florestal da UFV.

O convênio celebrado entre a Financiadora de Estudos e Projetos (FINEP) e o Governo de Minas Gerais - o INOVAMINAS - teve como objetivo construir a estrutura física de instalação do Polo de Excelência em Florestas, compreendendo o escritório da Unidade Gestora, a implantação física e o aparelhamento da Biblioteca Virtual Florestal e do Centro de Inteligência em Florestas (CIFlorestas), incluindo um auditório, sala de reuniões e de videoconferência (MINAS GERAIS, 2010). Conforme constatado, os recursos destinados à criação da Biblioteca Virtual e do CIFlorestas tiveram suas devidas destinações enquanto o recurso para construção da sede encontra-se parado, aguardando os trâmites burocráticos para sua construção na UFV.

Os dois últimos projetos de manutenção/consolidação/gestão auxiliam no desenvolvimento das atividades cotidianas a que o Polo se propôs, sendo que o último passava por processo de aprovação dos recursos para o período 2013-2015. 
Para elaborar e desenvolver projetos, o Polo precisa estar em contato com o setor privado, as universidades, secretarias de Estado e sociedade civil, atendendo às demandas para atuar como catalisador. Observou-se que o Polo se empenhou em iniciativas de divulgação de suas atividades, por meio de marketing institucional e participação em eventos, como também em reuniões com atores-chave. Assim, o Polo buscou estreitar os relacionamentos com os atores participantes através das reuniões do Comitê Gestor, que aconteceram pelo menos duas vezes ao ano.

No ambiente virtual, criou um espaço para discussão em uma rede social, denominada Rede de Comunicação do Polo de Excelência em Florestas, que possuía 347 membros, sendo que menos de 190 deles estão inscritos em grupos de discussão, além do fato de que as participações encontram-se paradas, visto que há grupos de discussão em que a última postagem aconteceu em fevereiro de 2010.

Adicionalmente, propôs a elaboração de um curso à distância para atualização profissional de técnicos e engenheiros da área de ciências agrárias, em parceria com a Coordenação de Ensino a Distância (CEAD) da UFV.

Finalizando, a execução das atividades de gestão do Polo exigiu que um grupo de profissionais desenvolvessem determinadas habilidades. Assim, 15 profissionais que passaram pelos cargos de consultores, secretárias e estagiários receberam treinamentos. Em razão do pagamento desses profissionais acontecer via bolsa, muitos deles acabam deixando a organização, que aumenta a rotatividade e os gastos com treinamento.

Em relação ao conjunto de projetos destinados à gestão do Polo, observou-se que em sua maioria, pelo menos em registros documentais, não há uma descontinuidade de projetos, sendo um substituído pelo outro, em sequência. Essa exceção aconteceu com o último projeto de gestão, que findou em junho (2013) e, mesmo com recursos garantidos, ainda aguardava liberação para o biênio 2013-2015.

\subsubsection{Sistema de Informação}

Pensando na sociedade e nos atores do setor florestal, o Polo de Excelência em Florestas desenvolveu o projeto de divulgação de dados e informações sobre a cadeia de base florestal, denominado "Sistema de Informação Florestal". Este tem como objetivo "compilar, produzir e disseminar informações técnicas, científicas, mercadológicas e conjunturais de interesse do sistema agroindustrial de base florestal” (MINAS GERAIS, 2013).

O projeto iniciou-se em 2008, com a aprovação do INOVAMINAS e se fortaleceu com a aprovação dos recursos do "Projeto de Operação e Manutenção do Centro de Inteligência em Florestas". Este segundo tinha como objetivo atender apenas ao Centro de Inteligência em Florestas (CIFlorestas), sendo posteriormente ampliado para atender também à Biblioteca Virtual Florestal e 
ao Bureau de Inteligência Florestal, componentes do Sistema de Informação Florestal (MINAS GERAIS, 2013).

O CIFlorestas é um site de informações do setor florestal com a finalidade de "subsidiar iniciativas públicas e privadas de apoio, de desenvolvimento sustentável e de Investimentos no setor" além de propor-se a ser referência ao divulgar e veicular informações e notícias sobre o agronegócio florestal (CIFLORESTAS, 2013). Em abril de 2013 o site atingiu a marca de mais de quatro mil acessos diários, sendo os três maiores acessos espontâneos internacionais localizados nos Estados Unidos, Portugal e Argentina (CIFLORESTAS, 2013).

Dentro do site do CIFlorestas é possível encontrar o Bureau de Inteligência, que contém: a análise conjuntural, editais, patentes depositadas, legislação, produção técnica e uma cartilha florestal. Além disto, o site conta com links de acesso às redes sociais, como o Facebook e o Twitter. Já a Biblioteca Florestal é um repositório digital da produção bibliográfica no campo das ciências florestais.

Em ata de reunião de apresentação das atividades, realizada em Viçosa em 2010, o então secretário da SECTES, Alberto Portugal, destacou a relevância desses instrumentos para dar visibilidade ao Polo nos cenários nacional e internacional.

No tocante aos recursos, foram investidos mais de $\mathrm{R} \$ 814.432,00$, visto que houve uma complementação prevista no projeto financiado pela FINEP. Além disso, percebe-se que na troca de um projeto e outro não houve uma continuidade favorável, uma vez que os recursos foram estimados apenas até junho, o que poderia comprometer as atividades do projeto e também dos bolsistas.

\subsubsection{Projetos de pesquisas em geral}

Dos projetos desenvolvidos pelo Polo, 11 foram classificados como projetos de pesquisas em geral, totalizando $\mathrm{R}$ \$3.237.962,00 em investimentos. Do total, três projetos têm objetivos de desenvolver tecnicamente o campo, porém sem uma tecnológica potencial de inovação selecionada. Tais projetos são importantes para identificar possíveis inovações no futuro, uma vez que as pesquisas estão em desenvolvimento e em nível mais básico. Outros três projetos têm caráter metodológico e de indicadores, outros três, de caracterização e diagnósticos setoriais e os dois últimos são de estruturação do Polo.

Relacionando os projetos de pesquisa em geral desenvolvidos com o processo de inovação, é possível identificar uma relação com a geração de ideias. Esse tipo de pesquisa é importante e deve acontecer constantemente na busca de novas oportunidades de inovação.

\subsubsection{Pesquisa específica com enfoque em inovação}


Os projetos desenvolvidos caracterizados como pesquisa específica com enfoque em inovação totalizaram $\mathrm{R} \$ 3.466 .602,52$, divididos em quatro projetos. Esses projetos foram classificados dessa maneira porque já havia uma tecnologia específica selecionada, com caráter inovador.

Por meio da análise de pequeno trecho dos resultados encontrados em relatórios do Polo foi possível identificar similaridades. Os projetos dos professores da UFV José Silva e Angélica Carneiro referiam-se ao termo transferência de tecnologia, enquanto os projetos dos professores Jorge Colodette e Sergio Motoike, a pedidos de patentes.

De acordo com o Instituto Nacional de Propriedade Industrial ([s.d.]) são formas de tecnologia as patentes; o desenho industrial, segredo industrial (know how); serviços de assistência técnica; e marcas e franquias. Para ter acesso a essas tecnologias existem três formas: por cessão, quando há transferência de titularidade do direito de propriedade intelectual; por licenciamento, quando se tem o uso do direito de propriedade intelectual de forma exclusiva ou não; e por transferência de tecnologia, quando há o fornecimento de informações não amparadas por direitos de propriedade industrial e serviços de assistência técnica (INSTITUTO NACIONAL DE PROPRIEDADE INDUSTRIAL, [s.d.]).

Assim, em dois projetos, a patente é um direito de propriedade sobre uma invenção enquanto a transferência de tecnologia, nos outros dois projetos, diz respeito a um segredo industrial (know how).

Em relação à participação do Polo nas pesquisas dos projetos que geraram o pedido de patente, acredita-se que o financiamento e o apoio ocorreram em uma fase de amadurecimento da ideia, já que os pesquisadores atuam nesses segmentos há muitos anos, caracterizando o processo como fruto de pesquisa básica.

Esses processos serão considerados inovação se chegarem à fase de mercado, ultrapassando os estudos científicos. Concluindo, os projetos desenvolvidos com pesquisa científica com enfoque em inovação podem ser considerados em fase de seleção do processo de inovação.

\subsection{Projetos propostos}

Em relação aos projetos propostos, nenhum deles faz referência à gestão do Polo nem ao Sistema de Informação Florestal, estando presentes outros tipos.

\subsubsection{Projetos de pesquisas em geral}

Três projetos dizem respeito a projetos de pesquisa em geral, com o enfoque no desenvolvimento de tecnologias, porém sem a identificação de uma inovação presente. Outro ponto a ser identificado é a ausência de projetos de diagnósticos setoriais e caracterização da cadeia 
produtiva, fruto da maturidade dos projetos concluídos graças ao ganho de conhecimento sobre o cenário.

Outros dois projetos são propostos com fins de desenvolver e validar metodologias e indicadores. Ambos propostos pelo mesmo pesquisador, sendo também desdobramentos de pesquisas passadas com o objetivo de aprimoramento de resultados.

Os outros dois projetos têm como objetivo capacitar agentes ligados à cadeia produtiva florestal. Este objetivo alinha-se ao proposto pelo Polo de Excelência em Florestas e, até então havia desenvolvido apenas ações isoladas em relação à capacitação, propondo então um projeto maior e estruturado.

\subsubsection{Pesquisa específica com enfoque em inovação}

Cinco projetos foram apresentados à SECTES e aguardavam resposta até 2013. Eles foram classificados contendo o enfoque em inovação, e coincidentemente, são projetos dos mesmos professores que entraram com pedido de patente. Pela descrição dos objetivos, trata-se de pesquisas complementares ou de desdobramentos das pesquisas anteriores. Uma delas, do Professor Motoike, é uma sequência necessária e importante para a patente, já que se trata de uma manutenção das sementes no campo. Ciente disso, esse projeto já foi aprovado, porém ainda aguardava liberação de recursos.

\subsection{Possíveis Projetos}

No que diz respeito às ideias para projetos futuros, identifica-se uma continuidade no sentido dos projetos propostos. Observam-se quatro propostas de pesquisas alinhadas ao tema das patentes; uma de organização de um evento de divulgação de pesquisas concluídas; outra de capacitação; e, por fim, uma aplicada à produção, na busca da criação de um modelo de autossustentabilidade para a marcenaria da UFV.

\subsection{Publicações}

O Polo de Excelência em Florestas, a partir de suas atividades desenvolveu 6 livros, 16 boletins técnicos e 47 análises conjunturais. A publicação das pesquisas através desses três instrumentos é uma ação positiva, uma vez que a sociedade adquire o conhecimento científico desenvolvido a partir das pesquisas e passa a aplicá-lo na prática cotidiana.

\section{DISCUSSÃO}

Como limitação da pesquisa tem se o fato de ela ter como objeto o Polo de Excelência em si, como um arranjo organizacional, sem estender para os atores que dele participam, sejam coordenadores de projetos, sejam participantes do comitê gestor. Além disso, a falta de acesso ao conteúdo de todos os projetos, da proposta aos resultados, limitou a compreensão aprofundada de 
cada um, principalmente no que diz respeito à interação universidade-empresa e o sentido da demanda evidenciada.

Referindo-se ao processo de inovação foi possível identificar uma grande quantidade de projetos voltados para a geração das ideias (Figura 2). Poucos projetos têm em sua proposta uma pesquisa com foco consolidado em uma possível inovação, sendo o restante voltado para atender à pesquisa básica e à dinâmica do setor, como aqueles voltados para metodologias, indicadores e diagnósticos de cenários. Os projetos de pesquisa em geral, concentraram-se no quadrante "gerar", e os projetos de pesquisa específicos concentraram-se em "selecionar" e "implementar".

Essa proporção entre geração, seleção e implementação pode ser considerada normal graças à própria dinâmica do alcance da inovação e também à amplitude de objetivos do Polo, sendo o meio de sustentação das atividades os projetos de gestão do Polo.

Não obstante, ressalta-se que todas as ações juntas buscaram colaborar de alguma forma para o desenvolvimento setorial e econômico do setor florestal, cumprindo com sua finalidade.

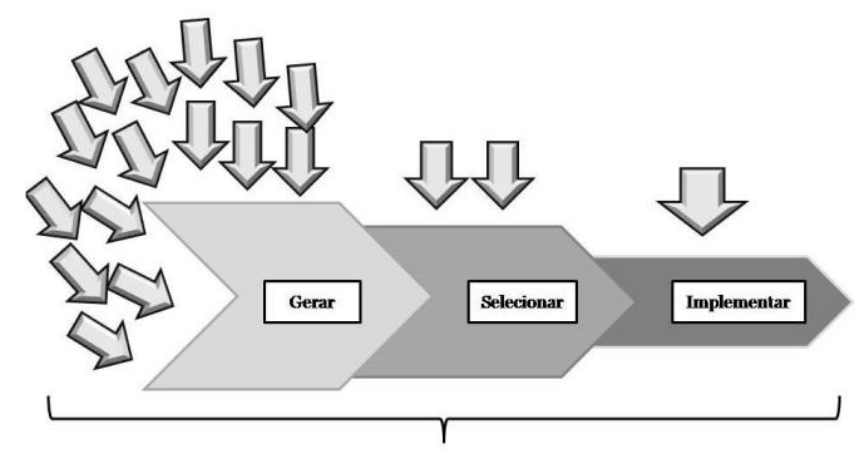

Figura 2 - Projetos do Polo sob a ótica do processo de inovação Fonte: Elaborado pela autora (2013)

Em relação à Hélice Tríplice, observou-se que o Polo de Excelência em Florestas caminha no sentido da inovação por uma articulação forte entre universidade e governo, com relações ainda tímidas com a iniciativa privada. Ainda assim, torna-se evidente nas propostas e projetos o princípio de atuação coletiva para alcançar a inovação, corroborando com o pensamento dos autores referenciados neste estudo.

Ao assumir o papel de catalisador de demandas do setor florestal e idealizador do Polo de Excelência em Florestas, por meio da SECTES, o Estado de Minas Gerais tem atuado de forma a preencher a lacuna entre o apoio universitário na formação de empresas e a aceitação por apoiadores financeiros de capital privado de risco.

\section{CONCLUSÃO}

Este trabalho apresentou uma análise das ações do Polo de Excelência em Florestas para a busca da inovação no setor florestal no estado de Minas Gerais. Esses dados podem servir de 
orientação para políticas públicas no direcionamento ao efetivo cumprimento dos objetivos dos Polos de Excelência. Contudo, a pesquisa também revela a escassez de dados e informações sistematizadas sobre o Polo de Excelência em Floresta e seus projetos, em que as informações são precárias e, por vezes, inexistentes.

A maior contribuição do Polo de Excelência em Florestas, até o momento, que o aproxima do alcance de seu objetivo de se tornar referência no setor florestal, refere-se ao projeto Sistema de Informação Florestal, responsável pela criação do Centro de Inteligência Florestal (CIFlorestas), da Biblioteca Virtual Florestal e do Bureau de Inteligência Florestal.

Como estudos futuros, sugerem-se pesquisas que possam aprofundar o entendimento no sistema de inovação mineiro e suas relações com o Polo de Excelência em Florestas, bem como conhecer sua dinâmica na percepção de outros atores, como as universidades, empresas do setor privado e associações civis ligadas ao setor florestal. Ademais, estudos que compreendam a situação de outros Polos podem ajudar na análise e no entendimento da política dos Polos de Excelência como um todo.

\section{AGRADECIMENTOS}

Ao Polo de Excelência em Florestas e a Secretaria de Estado de Ciência, Tecnologia e Ensino Superior de Minas Gerais (SECTES) pelas entrevistas e visitas, imprescindíveis à execução deste trabalho; e a Fundação de Amparo e Apoio a Pesquisa de Minas Gerais (FAPEMIG), pelo financiamento da pesquisa.

\section{REFERÊNCIAS}

BESSANT, J.; TIDD, J. Inovação e empreendedorismo. Traducao Elizamari Rodrigues Becker; Gabriela Perizzolo; Patrícia Lessa Flores Da Cunha. Porto Alegre: Bookman, 2009.

CASSIOLATO, J. E.; LASTRES, H. M. M. Sistemas de inovação e desenvolvimento: as implicações de política. São Paulo em Perspectiva, São Paulo, v. 19, n. 1, p. 34-45, 2005.

CIFLORESTAS. 5 anos do CIFlorestas, chegando a 4000 acessos diários. 2013. Disponível em: <http://www.ciflorestas.com.br/conteudo.php?id=8559>. Acesso em 2 de outubro de 2013.

EDQUIST, C. Systems of Innovations: Perspectives and Challenges. In: FAGERBERG, J.; ETZKOWITZ, H. The Triple Helix of university-industry-government: implications for policy and evaluation. Sister, Stockholm, 2002.

ETZKOWITZ, H. Hélice Típlice: Universidade-indústria-governo: inovação em movimento. Porto Alegre: Edipucrs, 2009.

FREEMAN, C. The "National System of Innovation" in historical perspective. Cambridge JournalofEconomics, Oxford, v. 19, n. 1, 1995.

INSTITUTO NACIONAL DE PROPRIEDADE INDUSTRIAL. Curso Básico em Propriedade Industrial: Nível Básico em Transferência de Tecnologia. INPI, [s.d.]. Disponível em: <http://unesp.br/nit/mostra_arq_multi.php?arquivo=5520>. 
MINAS GERAIS. Do Choque de Gestão ao Estado para Resultados.Os Marcos Referenciais do Planejamento Estratégico do Governo de Minas Gerais 2003-2010. Belo Horizonte, SEPLAG, 2011. Disponível

<http://www.agendademelhorias.org.br/uploads/documentos/registros_de_planejamento_estrategico .pdf>. Acesso em: 20 set. 2013

MINAS GERAIS. Minas Avança na economia do conhecimento. Inovação: uma face do Estado para Resultados. Belo Horizonte: SECTES, 2010.

MINAS GERAIS. Relatório de Atividades do Polo de Excelência em Florestas. Polo de Excelência em Florestas, Viçosa, 2013.

MINAS GERAIS. Relatório de Atividades do Polo de Excelência em Florestas. Polo de Excelência em Florestas, Viçosa, 2010.

ROSENBERG, N. Innovation and Economic Growth.OECD, 2004. Disponível em: <http://www.oecd.org/cfe/tourism/34267902.pdf>. Acesso em: 25 set. 2013.

SI-FLORESTAS. Biblioteca Virtual Florestal, 2013. Disponível em: <http://www.bibliotecaflorestal.ufv.br/>. Acesso em: 14 ago. 2013

SIMIONI, F. J.; BINOTTO, E.; HOFF, D. N. A inovação tecnológica na perspectiva dos gestores da Indústria madeireira da região de Lages, SC. Revista Árvore, Viçosa, v.36, n.5, p.981-987, 2012.

VALENTE, L. Hélice tríplice: metáfora dos anos 90 descreve bem o mais sustentável modelo de sistema de inovação. Conhecimento \& Inovação [online], v. 6, n. 1, p. 6-9, 2010. Disponível em: $<$ http://inovacao.scielo.br/scielo.php?script=sci_arttext\&pid=S1984-

$43952010000100002 \& \operatorname{lng}=$ pt\&nrm=is $>$. Acesso em 18 set. 2013.

Recebido: 28/02/2015

Aprovado: 02/04/2016 